Philosophie ANTIQUE

\section{Philosophie antique}

Problèmes, Renaissances, Usages

19 | 2019

L'épicurisme antique

\title{
Andrea FALCON \& David LEFEBVRE (éd.), Aristotle's Generation of Animals : A Critical Guide
}

Cambridge / New York, Cambridge University Press, 2018 (Cambridge Critical Guides), xiv-288 p., ISBN 978-1-107-13293-1

\section{Claire Louguet}

\section{(2) OpenEdition}

\section{Journals}

Édition électronique

URL : https://journals.openedition.org/philosant/2003

DOI : 10.4000/philosant.2003

ISSN : 2648-2789

Éditeur

Éditions Vrin

\section{Édition imprimée}

Date de publication : 31 octobre 2019

Pagination : 189-191

ISBN : 978-2-7574-2534-3

ISSN : $1634-4561$

\section{Référence électronique}

Claire Louguet, «Andrea falcon \& David Lefebvre (éd.), Aristotle's Generation of Animals : A Critical Guide», Philosophie antique [En ligne], 19 | 2019, mis en ligne le 16 mai 2019, consulté le 02 décembre 2022 URL : http://journals.openedition.org/philosant/2003; DOI : https://doi.org/10.4000/philosant.2003

\section{(c) (i) $(9)$}

Creative Commons - Attribution - Pas d'Utilisation Commerciale - Pas de Modification 4.0 International - CC BY-NC-ND 4.0

https://creativecommons.org/licenses/by-nc-nd/4.0/ 
Andrea Falcon \& David Lefebvre (éd.), Aristotle's Generation of Animals : A Critical Guide, Cambridge / New York, Cambridge University Press, 2018 (Cambridge Critical Guides), xiv-288 p., ISBN 978-1-107-13293-1.

Les treize articles publiés dans ce volume sont pour la plupart issus d'un colloque international sur la Génération des Animaux $(G A)$ organisé à la Sorbonne en 2014 par A. Gotthelf (dont le décès est survenu en 2013) et D. Lefebvre. Dans le contexte du « biological turn » initié il y a quarante ans dans les études aristotéliciennes, ce traité est l'objet, depuis quelques années, d'une attention accrue, comme en témoignent les nouvelles traductions - en français par D. Lefebvre en 2014 ; en anglais par A. Gotthelf et A. Falcon, en préparation -, la publication de la monographie de S. Connell Aristotle on Female Animals: A Study of the $G A$, Cambridge, 2016, ainsi que nombre d'articles écrits par les spécialistes, dont la plupart ont également contribué au présent volume. Premier recueil d'articles jamais consacré à la $G A$, ce volume vient combler un manque évident.

Si les études présentées ici portent sur des sujets divers, il faut aussi souligner l'unité de l'ouvrage, qui mérite le nom de « guide critique » : il permet de prendre la mesure des recherches actuelles et des débats interprétatifs dont est l'objet la $G A$, traité biologique aux enjeux philosophiques nombreux (ainsi que le soulignent Falcon et Lefebvre dans l'introduction) ; le volume est traversé du début à la fin par des questions et des thèmes qui se font écho, sans que les désaccords entre les interprètes soient masqués (ce qui rend la lecture de l'ensemble stimulante); enfin, les textes cités font l'objet de lectures précises et claires et les débats interprétatifs sont bien explicités, ce qui devrait donner au lecteur (y compris non spécialiste) la possibilité d'entrer dans le vif du sujet. Le recueil s'organise en quatre parties : I. The Unity and Structure of Aristotle's $G A$; II. The Principles of Animal Generation Reconsidered ; III. Hybrids, Male and Female, Particular Forms, and Monsters ; IV. Methodology in Aristotle's $G A$.

I. The Unity and Structure of Aristotle's GA (articles 1 à 3)

A. Gotthelf et A. Falcon (« "One Long Argument”? The Unity of Aristotle's $G A »)$ proposent un parcours de la $G A$ livre par livre et justifient le plan adopté par Aristote, en montrant notamment comment les livres 4 et 5 contribuent à l'économie générale du traité dont l'objet - telle est la thèse défendue - ne se limite pas à l'embryologie mais est en fait la génération de l'organisme adulte (en tant qu'il traite de la nécessité matérielle, le livre 5 se distingue de la perspective téléologique des livres II à IV des Parties des animaux).

D. Lefebvre («Parts and Generation: The Prologue to the $G A$ and the Structure of the Treatise »), à la faveur d'une lecture minutieuse du prologue, insiste sur la spécificité de la $G A$ par rapport aux autres traités zoologiques et soutient que le prologue introduit non pas au seul livre 1 (contre Gotthelf) mais à l'ensemble du traité, composé de deux sections principales (l'une sur les parties génératrices et l'autre sur les principes de la génération).

M. Leunissen (« Order and Method in Aristotle's GA 2 ») présente la façon dont Aristote lie intimement l'ordre d'exposition à l'ordre naturel du développement des êtres vivants, lesquels sont examinés en suivant l'ordre de la scala naturae. Cette lecture des chapitres 2.1-6 s'arrête en particulier sur les difficultés et les questions méthodologiques soulevées par Aristote lui-même.

II. The Principles of Animal Generation Reconsidered (articles 4 à 7)

P. Pellegrin («What Is Aristotle's GA About? ») soutient que la théorie de la génération est un cas particulier de la théorie de la nutrition (et non l'inverse) : le mouvement générateur, comme le mouvement responsable de la croissance, est un cas 
particulier du mouvement au moyen duquel l'âme nutritive élabore les nutriments selon des rapports définis.

D. Henry (« Aristotle on Epigenesis: Two Senses of Epigenesis »), s'oppose à M. Leunissen - qui soutenait en 2013 que l'embryon est capable d'adapter en temps réel sa forme en réponse à des changements affectant son environnement -, en arguant qu'une telle adaptation impliquerait un choix rationnel; il soutient une version plus faible de l'épigénèse permettant de sauver la fixité des espèces (toutes les puissances impliquées dans le développement de l'embryon sont de type non rationnel et leur activation, le cas échéant, s'effectue par contact). Cet article donne le sentiment que la conception d'Aristote se situe en réalité entre ces deux interprétations opposées (il faudrait, pour poursuivre ce débat, préciser ce qu'on entend par « adaptation » et se demander si, comme le dit l'auteur, la forme correspond au phénotype).

M. Rashed (« A Latent Difficulty in Aristotle's Theory of Semen: The Homogeneous Nature of Semen and the Role of the Frothy Bubble »), après avoir établi que la semence est un type d'homéomère particulier - il s'agit d'un mélange au sens faible puisqu'il contient des bulles de souffle chaud -, s'intéresse à ces bulles elles-mêmes, dont Aristote ne dit rien explicitement, mais qui, selon M. R., seraient responsables, non seulement de la fécondation, mais aussi du nombre et de la taille des embryons. Pour construire son hypothèse, M. R. examine d'abord les textes du Timée dans lesquels Platon traite de bulles, et dont l'exploitation par Aristote est vraisemblable ; ensuite, M. R. montre ce que les bulles aristotéliciennes ont de spécifique : en s'appuyant sur des textes de Métaphysique Z, notamment, il propose une réflexion spéculative reposant sur la distinction faite par Aristote entre matière sensible et matière intelligible des sphères spéculative à plus d'un titre puisque jamais Aristote ne fait explicitement le lien proposé ici.

C. Cerami («Function and Instrument: Toward a New Criterion of the Scale of Being in Aristotle's $G A \gg$ ) soutient que le critère de classification des êtres vivants ne réside pas pour Aristote dans les différences de chaleur (en quantité et en qualité), contre Gotthelf et Balme, mais dans leur capacité d'accomplir la fonction commune à tous les êtres, à savoir la capacité de persister en acte, c'est-à-dire, dans le cas des êtres vivants, la capacité de se reproduire (la chaleur vitale n'est qu'un instrument en vue de cette fin).

\section{Hybrids, Male and Female, Particular Forms, and Monsters (articles 8 à 11)}

J. Groisard (« Hybridity and Sterility in Aristotle's $G A \gg$ ), en proposant une lecture détaillée des chapitres 2.7-8 où Aristote débat avec Démocrite et Empédocle à propos de la stérilité des mules, montre que l'hybridité est chez Aristote une exception qui ne remet pas en cause la théorie générale de l'éternité des espèces et que, au sein de cette exception, le cas de la stérilité des mules fait elle-même figure d'exception.

J. Gelber (« Females in Aristotle's Embryology »), critique l'interprétation traditionnelle (la génération d'une femelle est un accident du processus optimal de génération qui devrait aboutir à un mâle) ainsi que l'interprétation de D. Henry (le sexe est déterminé uniquement par des facteurs matériels et la génération de femelles ne constitue pas un échec). Elle objecte à la première interprétation le fait qu'Aristote dit que la génération des femelles a une finalité et à la seconde qu'il existe bien une asymétrie selon lui entre mâle et femelle, et elle propose donc une troisième solution qui combine nécessité matérielle et finalité tout en tenant compte de l'asymétrie entre mâle et femelle.

G. Salmieri (« Something(s) in the Way(s) He Moves: Reconsidering the Embryological Argument for Particular Forms in Aristotle ») prend position dans le débat opposant les chercheurs depuis plusieurs années concernant la forme transmise 
par le mâle : celle-ci inclut-elle les traits individuels ? L'auteur propose une solution de compromis : ce qui est transmis par le mâle est une forme individuelle constituant une unité, au sein de laquelle on peut distinguer conceptuellement deux niveaux, la forme et les traits individuels, bien que l'une et les autres ne soient pas imputables à des facteurs distincts du côté du géniteur.

S. Connell (« Aristotle's Explanations of Monstrous Births and Deformities in GA $4.4 \gg)$ montre ce qu'il y a de scientifique dans la recherche d'Aristote sur les monstres : bien que ceux-ci ne relèvent pas de ce qui est conforme à la nature, on peut déterminer scientifiquement dans quels cas les monstres et difformités se produisent le plus souvent, ce qui nécessite une connaissance des différentes espèces et de leurs caractéristiques essentielles.

\section{Methodology in Aristotle's GA (articles 12 et 13)}

R. Bolton (« The Search for Principles in Aristotle: Posterior Analytics 2 and $G A 1$ ) s'oppose à une lecture communément admise (《three-stage account ») concernant la façon dont la recherche scientifique adéquate mènerait selon Aristote à la découverte de l'essence et de la définition (signification du nom, découverte de l'existence, découverte de l'essence). R. B. examine un à un tous les textes des Analytiques sur lesquels s'appuie cette lecture pour montrer dans chaque cas qu'elle ne s'impose pas. Il soutient au contraire que l'existence est donnée et trouve dans $G A 1$ la confirmation de son hypothèse.

J. Lennox («Aristotle, Dissection, and Generation: Experience, Expertise, and the Practices of Knowing ») propose une lecture fine de tous les passages de la $G A$ faisant référence aux anatomai et se demande à chaque fois si le terme désigne un schéma ou une dissection et quel type d'expertise requièrent leur production et leur observation.

Nul doute que ce volume fera date et contribuera, non seulement à nourrir les débats en cours, mais aussi à convaincre les lecteurs qui l'ignoreraient encore que la biologie aristotélicienne est proprement philosophique.

Claire Louguet

UMR Savoirs, Textes, Langage (CNRS / Univ. Lille)

Diana Quarantotto, L'universo senza spazio. Aristotele e la teoria del luogo, Napoli, Bibliopolis, 2017 (Elenchos, 64), 279 p., ISBN 978-88-7088-648-1.

L'ouvrage se présente comme un commentaire continu des chapitres de la Physique d'Aristote consacrés à l'étude du lieu (IV, 1-5). Le texte grec est cité d'après l'édition de Ross, et traduit par l'auteure de manière toujours très précise et rigoureuse (si ce n'est l'introduction d'une négation indue à la ligne 212a31, p. 237). L'ensemble permet de se donner une bonne conception de l'importance qu'Aristote accorde au lieu pour expliquer le mouvement des corps, de sa différence avec la notion d'espace, et de son inscription dans un univers considéré comme plein et continu.

Le découpage en courts passages fait apparaître les articulations du raisonnement, qui progresse suivant la méthode habituelle d'Aristote dans ces matières : justification de l'étude du lieu dans le cadre de la physique ; raisons courantes pour lesquelles on peut soupçonner son existence; difficultés liées à son existence; difficultés à caractériser son essence ; mise à l'épreuve des différentes définitions possibles et sélection de la seule valide, qui permet de répondre aux difficultés soulevées au départ. Pour chaque extrait, l'auteure reconstitue très didactiquement les arguments, signalant les prémisses implicites, vérifiant la validité de chacune ainsi que de la conclusion. L'avantage de 